\title{
Mix Your Own Data Cocktail Served with A Slice of Adobe
}

\author{
Mark Wigley \\ Esri Switzerland,m.wigley@esri.ch
}

Keywords: Map Production and Geoinformation Management, Map Design, Cartography for Leisure

\begin{abstract}
:
Many cartographers, both professional and amateur, often struggle with finding reliable and freely available data for their cartographic projects.

One major source of such data is Esri's ArcGIS Living Atlas of the World which is one if not the foremost collection of geographic information from around the globe. This extensive repository contains thousands of maps, applications, and data layers.

Until recently this collection was only available to those using the Esri System. Esri has now however build a bridge to Adobe's Creative Cloud products Photoshop and Illustrator in the form of a free extension. This extension now allows users of these products to tap into this rich source of reliable geographic data, quickly and easily without leaving the comfort of the application.

In this presentation I will show how using Adobe's Illustrator and Photoshop one can easily access this data, mix it with other external files in various formats and generate POIs (Points of Interest) from both addresses and names. I will also show how the imported layers can be modified, the design changed, labels added, and filters used. Furthermore, I will demonstrate the available analysis tools such as those to create routes or calculate a buffer using travel times.
\end{abstract}

